\title{
An unusual cause of chronic renal dysfunction
}

\author{
Maria do Mar Menezes ${ }^{1}$, Patrícia Alves ${ }^{1}$, Aníbal Ferreira ${ }^{1}$, Helena Sousa ${ }^{1,2}$, Mário Góis ${ }^{1,2}$, Fernando Nolasco $^{1}$ \\ ${ }^{1}$ Department of Nephrology, Hospital Curry Cabral, Centro Hospitalar Universitário de Lisboa Central \\ 2 Laboratory of Renal Morphology, Centro Hospitalar de Lisboa Central - Hospital Curry Cabral, Lisboa, Portugal
}

\section{CLINICAL PRESENTATION}

We present the case of a 41-year-old caucasian woman with past medical history of pre-eclampsia, obesity, cholecystectomy, goiter and chronic kidney disease of unknown etiology diagnosed at age of 21. She began peritoneal dialysis at 23 years of age and transitioned to hemodialysis 3 years later due to failure of the technique.

At the age of 29, she underwent deceased donor kidney transplantation with 3 HLA-mismatches, a panel-reactive antibody of $80 \%$ and a cold ischemia time of 14 hours, with a antithymocyte globulin induction immunosuppression regimen. Both the donor and recipient tested positive for IgG anti-cytomegalovirus.

Due to hypersensibilization the patient was also treated with rituximab and IV immunoglobulin. The immediate postoperative period was uneventful. She was discharged after 40 days with the following maintenance immunosuppression: tacrolimus, mycophenolate mofetil, and steroids; with a serum creatinine $[\mathrm{s} C \mathrm{r}]$ of $1.9 \mathrm{mg} / \mathrm{dL}$ and no proteinuria. The protocol renal allograft biopsies (one week and one month after transplant) did not reveal any rejection criteria nor other alterations.

In the post-transplant period, there was the need to change the immunosuppressive therapy from mycophenolate mofetil to azathioprine due to her wish to become pregnant.

\section{Figure 1}

Jones Methenamine, 200X

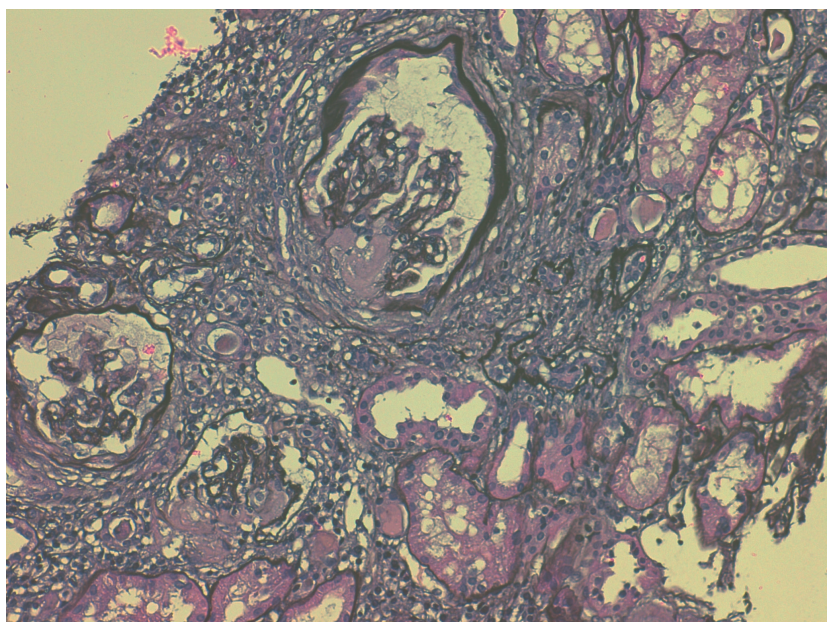

In the following years there were numerous episodes of urinary tract infections (UTIs), none of them requiring admission to the hospital and with moderate impact on renal function with sCr oscillating between $1.6 \mathrm{mg} / \mathrm{dl}$ and $2.6 \mathrm{mg} / \mathrm{dl}$. Prophylactic antimicrobial agents were begun to prevent UTIs, resulting in a significant decrease in their frequency.

There was a persistently elevated serum uric acid (averaging 10 $\mathrm{mg} / \mathrm{dl}$ ) with several gout crises and interphalangeal articular deformation, with small nodules at palpation. The initiation of allopurinol was considered, but contraindicated in association with azathioprine due to a risk of bone marrow suppression.

Ten years after the renal transplant, she developed mild proteinuria $(531 \mathrm{mg} /$ day) and progressive worsening of renal graft function $(\mathrm{sCr}$ $3.1 \mathrm{mg} / \mathrm{dl})$.

The presence of a subnephrotic proteinuria and a renal function aggravation can be caused by a late rejection, "de novo glomerulonephritis", or an ongoing infection, among others. The nature of the original renal disease is not known; therefore a recurrence disease is impossible to diagnose for sure. Based on clinical and laboratory findings alone it was not possible to reach a definite diagnosis.

A renal allograft biopsy was performed; images of histology sections are presented below.

\section{Figure 2}

Masson's Trichrome, 400X

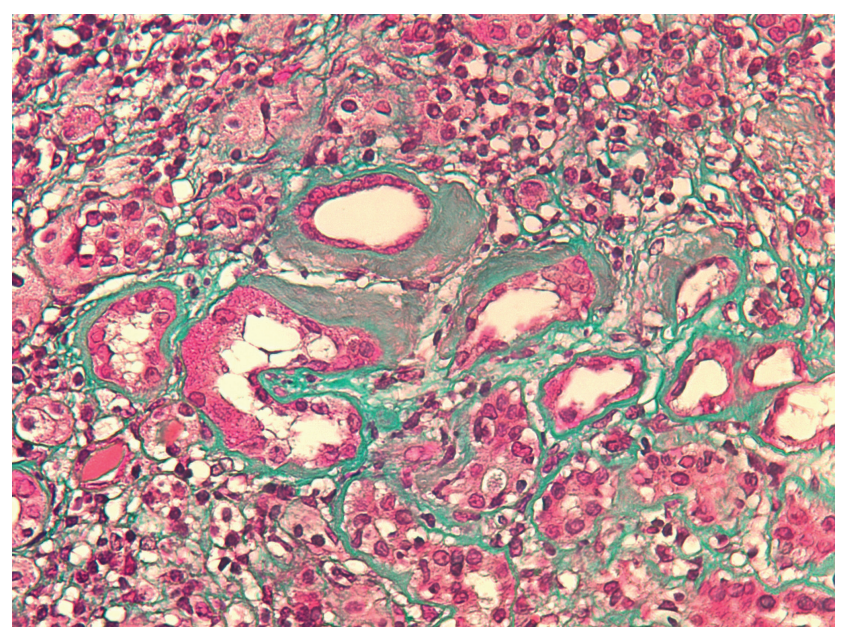




\section{Figure 3}

Periodic acid shift, $400 \mathrm{X}$

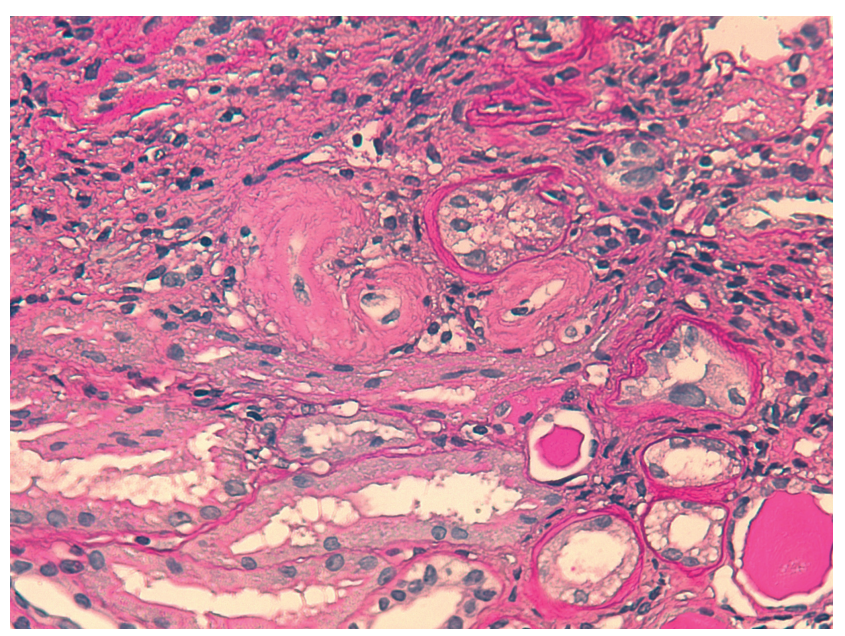

\section{Figure 4}

Congo Red, 200X

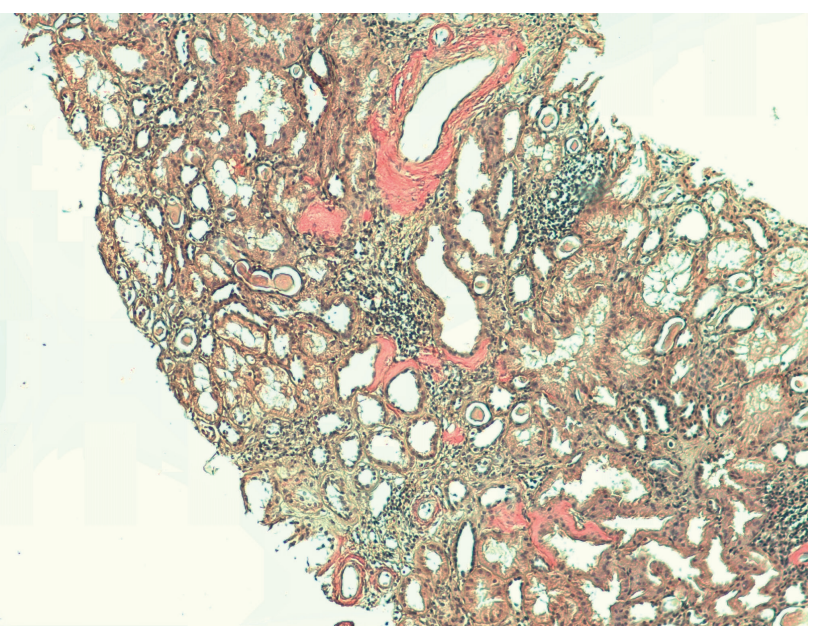

\section{QUESTIONS}

1. According to the clinical, laboratory, histologic and immunofluorescence findings, what is the most likely diagnosis?

2. Was the diagnosis of AA amyloidosis expected? What is the appropriate next step in patient evaluation?

3. What is an appropriate course of treatment and what is the prognosis in this patient?

\section{Figure 5}

Immunofluorescence - Amyloid A protein, 200X

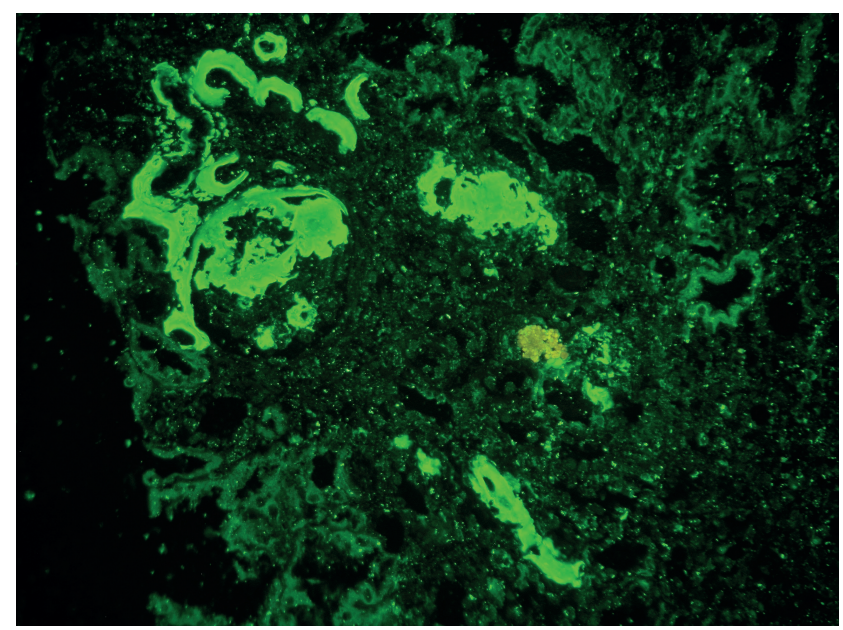

\section{Figure 6}

Immunofluorescence - A substance, 400X

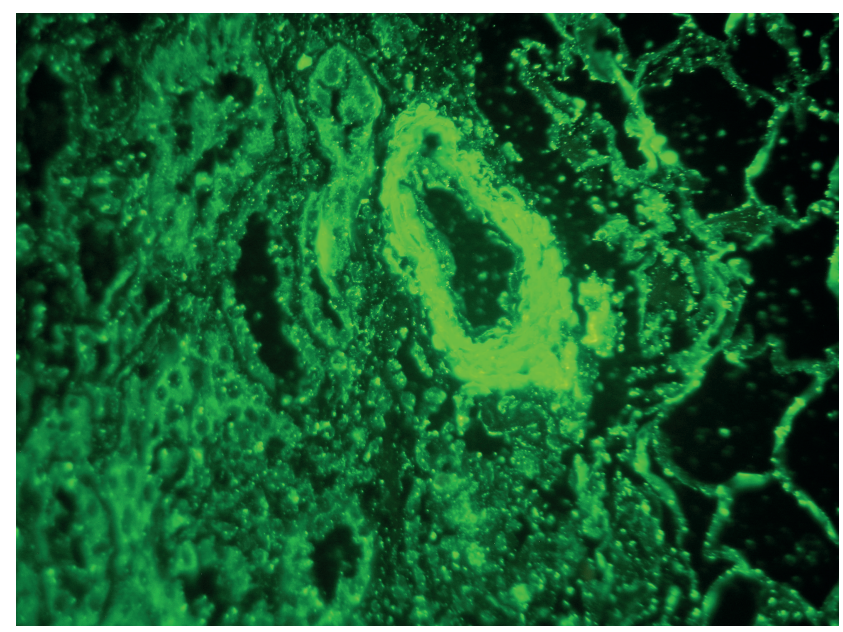

\section{ANSWERS}

1. According to the clinical, laboratory, histologic and immunofluorescence findings, what is the most likely diagnosis?

The biopsy shows subcapsular renal cortical with 9 glomeruli and several arteries.

Deposition of a low argyrophilic substance i.e. reduction of Jones silver coloration, is observed in glomeruli in Figure 1. 
Deposition of an amorphous substance is also observed in vascular walls (Figure 2) and tubular basement membrane (Figure 3).

The cortical fragment presents $40 \%$ interstitial fibrosis.

In Figure 4 the vascular walls stained orange by Congo red coloration. The observation under polarized light reveals apple-green birefringence consistent with amyloid deposition.

We made the identification of amyloid protein by immunofluorescence. We looked for kappa and lambda light chain and A protein. In Figure 5 we observed positivity for SAA (serum amyloid A protein) protein in glomerular tufts, basal tubular membranes and vascular walls.

These findings are in favor of AA amyloidosis in a renal allograft.

\section{Was the diagnosis of AA amyloidosis expected? What is the appro- priate next step in patient evaluation?}

AA amyloidosis is a disease of extracellular deposition of amyloid A protein in the form of fibrils assembled in a $\beta$-pleated structure in one or more organs of the body leading to progressive organ dysfunction, with the kidneys, liver and spleen being the main target organs of AA amyloid deposits. It is a long-term complication of several chronic inflammatory disorders such as chronic infections (tuberculosis, osteomyelitis, chronic pyelonephritis), rheumatologic disorders (ankylosing spondylitis, rheumatoid arthritis, psoriatic arthritis), inflammatory bowel diseases (Crohn's disease, ulcerative colitis) hereditary autoinflammatory diseases, malignancies and diseases predisposing to recurrent infections ${ }^{1}$.

Recurrent AA amyloidosis in the renal allograft has been documented in patients with tuberculosis, chronic pyelonephritis, familial Mediterranean fever and rheumatologic diseases such as ankylosing spondylitis and rheumatoid arthritis; however, de novo amyloidosis is rarely described ${ }^{2-4}$.

Signs of renal allograft amyloidosis are usually proteinuria and elevation in serum creatinine; presentation may be acute with nephrotic syndrome or very insidious, as it arose in our patient with mild proteinuria. The patient had no obvious signs or symptoms suggesting the presence of any of the diseases typically associated with AA amyloidosis, making the histologic findings a surprise. From this point, the next appropriate step would be to perform adequate investigation to exclude underlying chronic inflammatory diseases and infections.

Further studies revealed mildly elevated C-reactive protein (4 $\mathrm{mg} / \mathrm{dl}$ ) and erythrocyte sedimentation rate $(52 \mathrm{~mm})$, normal serum protein electrophoresis, elevated free light chains (free light kappa chain $137 \mathrm{mg} / \mathrm{L}$ and free light lambda chain $44.10 \mathrm{mg} / \mathrm{dL}$ ) but with a normal ratio kappa/Lambda and no serum monoclonal components. The complement system study showed no abnormalities apart from hypocomplementemia C3 (0.80 g/L). Autoimmunity tests (antinuclear, double-strand anti-DNA, extractable nuclear antigens, anticitrullinated protein antibody and rheumatoid factor) were all normal. Infectious serology for hepatitis B and C virus, HIV, cytomegalovirus and polyomavirus BK were negative. Negative results were obtained for urine cultures. Serum amyloid $A$ was above normal range $(27.1 \mathrm{mg} / \mathrm{L})$. Annual exams including pelvic, abdominal, thyroid, vesical and renal ultrasonography with Doppler revealed no abnormalities.

However, there is one important element in this case that must not be overlooked, the recurrent gout crises. To the present date, there are 13 published cases describing an association between gout and amyloidosis; all patients had chronic tophaceous gout for at least 10 years and did not use colchicine regularly ${ }^{5}$. The exact pathophysiological mechanism for the association between gout and amyloidosis is not entirely understood, but is thought to be linked to the persistent elevation of acute-phase reactants. A hypothesis that may explain a much lower incidence of amyloidosis in association with gout, compared to other rheumatologic disorders such as rheumatoid arthritis, is the different inflammation pattern (it is usually short, intense and self-limiting) and the use of colchicine, a drug that has shown to prevent casein-induced amyloidosis in mice ${ }^{6}$.

\section{What is an appropriate course of treatment and what is the prog- nosis in this patient?}

Treatment of AA amyloidosis is directed towards control of the underlying disease responsible for amyloid formation. Early diagnosis and rapid control of the underlying inflammatory disease are of utmost importance to prevent irreversible organ damage ${ }^{1}$.

The mean survival time of patients with AA amyloidosis is 133 months (higher than in AL amyloidosis), with infections and cardiovascular events being the main causes of early death. The prognosis is closely related to serum amyloid $A(S A A)$ levels; the relative risk for death for patients with an SAA concentration below $4 \mathrm{mg} / \mathrm{l}$ is almost 18 times lower than in those with an SAA concentration of $155 \mathrm{mg} / \mathrm{l}$ or greater ${ }^{7}$. Thus, aiming for this target is important; it is related to regression of amyloid deposits in $60 \%$ of patients and superior survival. Older age, end stage renal disease (ESRD) at baseline and reduced serum albumin concentration are other factors associated with increased mortality.

The long-term consequences of amyloid deposition on the renal allograft remain unknown, but experience reported in the literature suggests a fairly benign renal outcome in those patients with recurrent amyloid of the renal allograft 2,8 .

In the case described, although gout is a hypothesis, there is no clear underlying disease responsible for amyloid formation, and due to the lack of evidence for a specific inciting process, treatment options are limited.

Eprodisate, a compound aiming to prevent the formation of amyloid deposits, slowed decline of renal function in patients with AA amyloidosis. However, it did not affect amyloid levels, proteinuria, ESRD or mortality risk ${ }^{9}$. Thus, in addition to it not being available in Portugal, the results were not very robust and failed to confirm benefit. Other combinations of CPHPC and anti-SAP antibody, as well as new biologics, are currently under evaluation. 
A valid treatment option in this case would be to aim for strict control of serum acid uric levels and prevention of future gout crises, while closely monitoring renal allograft function and proteinuria. Since colchicine has also been shown to decrease amyloid formation in mice (as well as being a drug directed to gout treatment), it is also an option to be considered in this patient.

The immunosuppressive therapy in our patient was also changed from azathioprine to mycophenolate mofetil, and therefore, we were able to start allopurinol. Hence, the control of the underlying inflammatory disease is now easier to attain.

Disclosure of potential conflicts of interest: none declared.

Authors' Contributions: equal autorship of Maria do Mar Menezes and Patrícia Alves.

\section{References}

1. Obici L, Merlini G. AA amyloidosis: Basic knowledge, unmet needs and future treatments. Swiss Medical Weekly. 2012.

2. Harrison KL, Alpers CE, Davis CL. De Novo Amyloidosis in a Renal Allograft: A Case Report and Review of the Literature. Am J Kidney Dis. 1993;22(3):468-76.

3. Y.lmaz S, Özçakar ZB, Bulum B, Kiremitçi S, Ensari A, Ekim M, et al. De novo amyloidosis in a renal transplant patient. Pediatr Transplant. 2014 Dec 1;18(8):E259-61.

4. White WE, Olone EL, Sheaff MT, Yaqoob MM. Graft pyelonephritis causing graft failure from de novo AA amyloid. Kidney Int. Elsevier BV; 2014 Jan 31;85(2):481.

5. Ter Borg EJ, Wegewijs MA, De Bruin P. Gout and AA amyloidosis: A case report and review of the literature. Journal of Clinical Rheumatology. Lippincott Williams and Wilkins; 2017. p. 233-4.

6. Levo Y, Shalev O, Rosenmann E, Eliakim M. Gout and amyloidosis. Ann Rheum Dis. 1980;39(6):589-91.

7. Feehally J, Floege J, Tonelli M, Johnson RJ. Renal Amyloidosis and Glomerular Diseases With Monoclonal Immunoglobulin Deposition. In: Comprehensive clinical nephrology. 6th ed. 2019.

8. Rojas R, Josephson MA, Chang A, Meehan SM. AA amyloidosis in the renal allograft: A report of two cases and review of the literature. Clin Kidney J. Oxford University Press; 2012;5(2):146-9.

9. Dember L. Eprodisate for the Treatment of Renal Disease in AA Amyloidosis. N Engl J Med. 2007;392-3.

\section{Correspondence to:}

Helena Sousa, MD

Laboratory of Renal Morphology, Centro Hospitalar de Lisboa Central - Hospital Curry Cabral, Lisboa, Portugal

E-mail: viana.helena@gmail.com 